\title{
Reliability analysis of Cognitive Radio Networks with balking and reneging
}

\author{
Mohamed Hedi Zaghouani \\ Doctoral School of informatics \\ University of Debrecen \\ Debrecen,Hungary \\ zaghouani.hedi@inf.unideb.hu
}

\author{
Hamza Nemouchi \\ Doctoral School of informatics \\ University of Debrecen \\ Debrecen,Hungary \\ nemouchi.hamza@inf.unideb.hu
}

\author{
János Sztrik \\ Doctoral School of informatics \\ University of Debrecen \\ Debrecen,Hungary \\ sztrik.janos@inf.unideb.hu
}

\begin{abstract}
The principle of balking and reneging on Cognitive Radio Networks taking into consideration servers unreliability, is investigated in this paper. In today's networking environment, the concepts of balking and reneging are very common. The more crowded the system is, the more discouraged potential customers will be, on the other hand, once their total waiting time hits the maximum, impatient users exit the system. To be closer to reallife scenarios, servers unreliability is taken into consideration.
\end{abstract}

Index Terms-Finite source queuing systems, simulation, Cognitive Radio Networks, performance and reliability measures, non-reliable servers, balking and reneging.

\section{INTRODUCTION}

Customers' impatience is a crucial factor to consider while modeling call centers. Balking and reneging are two frequent ways for customers to express their impatience. A call-in customer who cannot be helped immediately by a human server might be told how long a wait he/she faces before an operator is available. The customer may then choose to hang up (i.e. balk) or wait. This is known as balking, which occurs when a customer refuses to enter a queue because it is too long. A consumer waiting for an operator, on the other hand, may hang up (i.e. renege) before being served if the line becomes too lengthy. This is the reneging behavior. We will be applying these feature on our system.

The primary aim of our "Cognitive Radio Network" model is to use the free spaces in the primary frequency band to support the secondary one. More details can be found in [1], [2], [3], [4], [5], and [6].

The network is made up of two main components. The first one is designed for Primary Users (PU) with a finite number of sources who produce primary calls after an exponentially distributed time. To be served, all of the created calls will be placed in a FIFO queue. The service time is distributed exponentially. The second subsystem is dedicated to the jobs of Secondary Users (SU), which are created from a limited number of sources and directed to the Secondary Channel Service (SCS) for processing. These calls have an exponential arrival time, but their service time is generally distributed using hypoexponential, hyper-exponential, and gamma distributions.

The created licensed calls will check the availability of the Primary Channel Service (PCS), if it is available, the service will begin immediately, if it is already in use by a primary call, the later call will be joining the FIFO queue. If the PCS is taken by a secondary customer, service will be interrupted immediately and the customer in question will be led back to the SCS. The aborted call would be restarted from the beginning of its operation or added to the retrial queue (orbit), depending on the current situation of the secondary service unit.

Secondary Channel Service SCS, on the other hand, receives requests that are not licensed. If the intended server is idle, SU is permitted to start the service, if it is occupied, they will attempt to start their service in the PCS in an opportunistic manner. If the last service channel is not occupied, the low priority call will be able to begin the service, otherwise, if it is occupied, the call will be immediately added to the orbit. Based on an exponential distributed time, concerned calls will retry to enter the server.

Several researchers have examined the CRN based on different scenarios. The effect of server unreliability on the $\mathrm{CRN}$, for example, was examined by the authors of [4], [11] and [12], however, balking and reneging were not taken in consideration by anyone of them. In [7], the same model was used taking into consideration abandonment, with SUs being required to quit the network when their cumulative patience time reaches a predetermined limit.

Balking and reneging have been applied on different retrial queuing systems, in [8], this theory was studied on the M/M/S retrial queue, and in [9] was added to an $\mathrm{M} / \mathrm{M} / 1 / \mathrm{N}$ retrial queue.

After a thorough search of many relevant topics and papers, we were unable to find any investigations that discussed this model in the case of balking and reneging assuming that the secondary model's server is unreliable, which is the novelty of our paper.

\section{SYSTEM MODEL}

The queuing cognitive radio system shown in Fig1 is based on the following assumptions. Consider two interconnected subsystems whose primary requests are created from a $N_{1}$ finite number of sources and forwarded to the first server after an exponentially dispersed period with a mean value of $1 / \lambda_{1}$. If the unit is available, the service will begin, otherwise, the call will be placed in the FIFO queue. The principal user's 


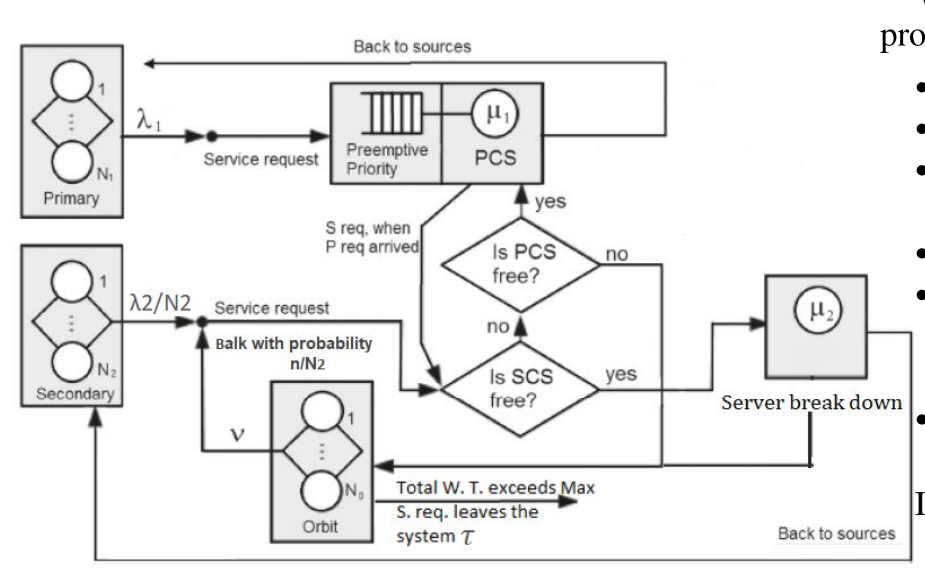

Figure 1. System model.

service time is an exponentially distributed random variable with the parameter $\mu_{1}$.

$N_{2}$ represents the secondary subsystem's number of sources. After an exponentially distributed interval of time, each source produces low priority jobs with parameter $\lambda_{2} / N_{2}$. The service time of SUs is generally distributed using hypo-exponential, gamma and hyper-exponential distributions, all of which have the same mean and different variances, with a rate $\mu_{2}$. The retrial time of the secondary customer is considered to be a random variable with an exponential distribution and a parameter of $\nu$.

With a probability of $n / N_{2}$, new arriving secondary customers may balk (refuse to join the server), where $n$ is the number of users in the network and $N_{2}$ is the number of sources. They may renege from the system (leave the orbit after joining) if service does not start by a given random time, which is exponentially distributed with parameter tau. Some breakdowns and repairs will appear unpredictably at the secondary server level based on exponentially distributed times with parameters $\gamma_{1}$ and $\gamma_{2}$, respectively. All the input parameters are collected in I.

Table I

INPUT PARAMETERS OF THE SIMULATION

\begin{tabular}{|c|c|c|}
\hline Parameters & The maximum & Value at time $t$ \\
\hline Active licensed sources & $N_{1}$ & $k_{1}(t)$ \\
\hline Active unlicensed sources & $N_{2}$ & $k_{2}(t)$ \\
\hline High priority arrival parameter & & $\lambda_{1}$ \\
\hline Low priority arrival parameter & & $\lambda_{2} / N_{2}$ \\
\hline Jobs in FIFO queue & $N_{1}-1$ & $q(t)$ \\
\hline Jobs in orbit & $N_{2}-1$ & $o(t)$ \\
\hline Primary service parameter & & $\mu_{1}$ \\
\hline Secondary service rate & & $\mu_{2}$ \\
\hline Reneging parameter & & $\tau$ \\
\hline Secondary server breakdown parameter & & $\gamma_{1}$ \\
\hline Secondary server repair parameter & & $\gamma_{2}$ \\
\hline Retrial rate & & $\nu$ \\
\hline
\end{tabular}

We suppose the below notations to model a stochastic process defining the functionality of the system:

- $k 1(t)$ : Number of primary jobs at given time $t$;

- $k 2(t)$ : Number of secondary jobs at given time $t$;

- $q(t)$ : The number of high-priority calls in the queue at time $t$

- $o(t)$ : Number of jobs in the orbit at time $t$;

- $y(t)=0$, if the PCS is idle, $Y(t)=1$, if PCS is occupied by a high-priority request and $Y(t)=2$, if the PCS is busy with a low-priority request at time $t$;

$c(t)=0$, if SCS is free and $c(t)=1$, if SCS is busy at time $t$.

It is easy to see that:

$$
\begin{gathered}
k_{1}(n)= \begin{cases}N_{1}-q(t), & y(t)=0,2 \\
N_{1}-q(t)-1 & y(t)=1\end{cases} \\
k_{2}(n)= \begin{cases}N_{2}-o(t)-c(t), & y(t)=0,1 \\
N_{2}-o(t)-c(t)-1 & y(t)=2\end{cases}
\end{gathered}
$$

\section{SimUlATION RESULTS}

The impact of service time distributions and the cognitive technology on our system's main performance measures are investigated in this section. SimPack [10] was used to create a stochastic simulation program in the $\mathrm{C}$ programming language. Except for the secondary service rate, all the network's random variables are considered to be exponentially distributed. All the numerical results were obtained by the validation of the simulation outputs. II lists the numerical values of the simulation main class input parameters, while III lists the numerical values of the simulation program's statistical class.

Table II

THE INPUT PARAMETERS OF SIMULATION

\begin{tabular}{|c|c|c|c|c|c|c|c|c|c|}
\hline$N_{1}$ & $N_{2}$ & $\lambda_{1}$ & $\lambda_{2} / N_{2}$ & $\mu_{1}$ & $\mu_{2}$ & $\nu$ & $\tau$ & $\gamma_{1}$ & $\gamma_{2}$ \\
\hline 25 & 50 & 0.2 & X-axis & 2 & 2 & 0.2 & 0.2 & 0.1 & 0.2 \\
\hline
\end{tabular}

Table III

PARAMETERS OF THE GENERAL DISTRIBUTIONS

\begin{tabular}{|c|c|c|c|c|}
\hline Distribution & Gamma, $c_{x}^{2}<1$ & Hyper & Hypo & Gamma, $c_{x}^{2}>1$ \\
\hline Parameters & $\alpha=1,7857 \beta=1,7857$ & $\begin{array}{c}p=0,3309 \\
\lambda_{1}=0,66198 \\
\lambda_{2}=1,33803\end{array}$ & $\begin{array}{c}\lambda_{1}=1,4854 \\
\lambda_{2}=3,06\end{array}$ & $\begin{array}{c}\alpha=0,3906 \\
\beta=0,3906\end{array}$ \\
\hline Mean & 1 & 1 & 1 & 1 \\
\hline Variance & 0.56 & 2.56 & 0.56 & 2.56 \\
\hline$c_{x}^{2}$ & 0.56 & 2.56 & 0.56 & 2.56 \\
\hline
\end{tabular}

1) Service times are generally distributed: Figure 2 shows how the distribution of primary and secondary service times affects the mean residence time of SUs vs the generation of secondary request times. When service times are gamma distributed with a $C_{x}^{2}>1$, a significant distributions sensitivity can be observed. The same clear sensitivity is seen in Figure 3, where the effect of primary and secondary service times distribution on the mean reneging time of SUs vs secondary request time generation was displayed, while gamma with a $c_{x}^{2}$ greater than one. Figure 4 confirms the 


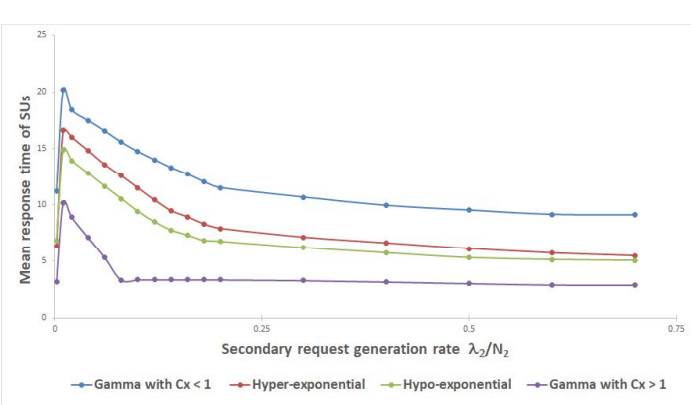

Figure 2. The impact of primary and secondary service times distribution on the mean residence time of SUs vs secondary request time generation

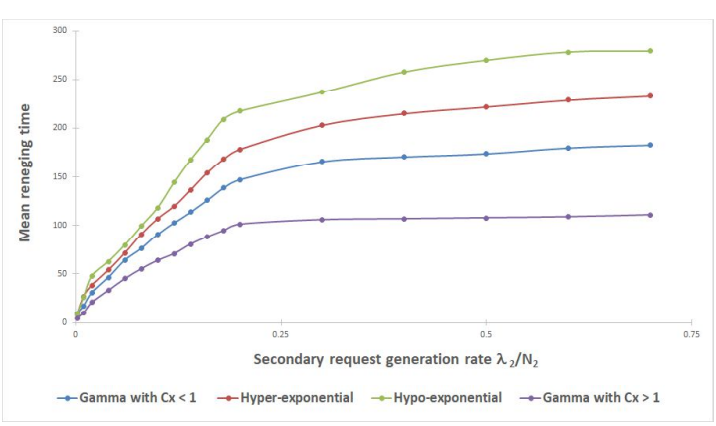

Figure 3. The impact of primary and secondary service times distribution on the mean reneging time of SUs vs secondary request time generation

similar behaviour shown in the previous two figures. Using the gamma distribution, which has a $C_{x}^{2}>1$. Furthermore, as expected, increasing the SU arrival intensity leads to more balking rate, we note as well a significant number of customers balk from the system.

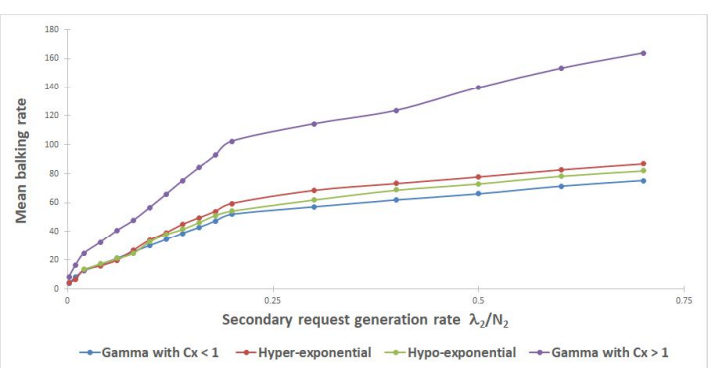

Figure 4. The effect of primary and secondary service times distribution on the mean balking rate vs secondary request time generation

2) All inter-event time are exponentially distributed: In this section, we suppose that all inter-event times are exponentially distributed. Same value of the parameters shown in I are applied with $\lambda_{2}=0.5$. We'd like to look into the effect of conductivity on the system's properties.

Figure 5 depicts the effect of increasing $N_{2}$ on the primary arrival rate and the number of sources on the mean response time of cognitive customers. In this graph, we can see that the primary arrival intensity has a significant impact on the average residency time of SUs, as when $\lambda_{1}=\lambda_{2} / 2$, the results show a smaller value of the mean than when $\lambda_{1}=\lambda_{2} * 2$.

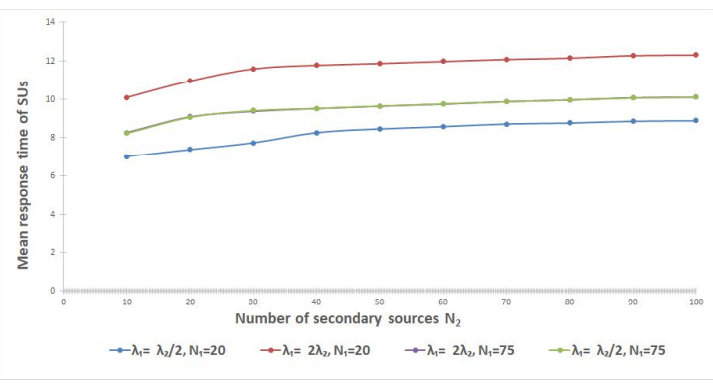

Figure 5. The effect of the primary network parameters on the average response time of SUs vs $N_{2}$

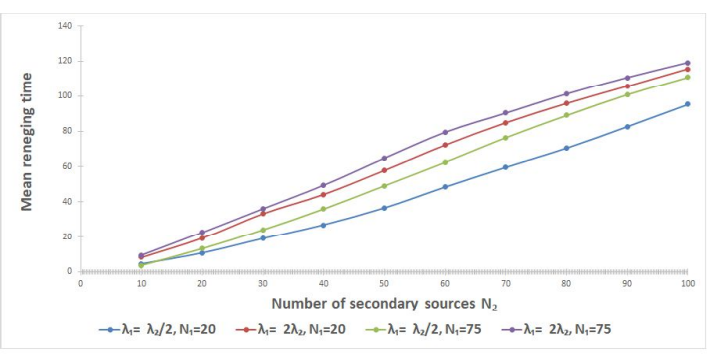

Figure 6. The effect of the primary network parameters on the mean reneging time of SUs vs $N 2$

In contrast to the primary number of sources, which has no effect, as when $N_{2}$ is high the traffic intensity in the primary sub-subsystem is bigger. However, other effects can be shown in Figure 6 when the primary number of sources is bigger. This is due to more secondary customers are reneging from the system.

Figure 7 illustrates the impact of $N_{2}$ on the mean balking rate within different configurations in the primary network. The only effect that can be seen is when the primary interarrival parameter is half the secondary arrival intensity. In this case, fewer customers do not enter the system. However, increasing $\lambda_{1}$ or $N_{2}$ there is almost no effect on the mean balking rate. As expected, increasing $N_{2}$ involves a greater mean balking rate.

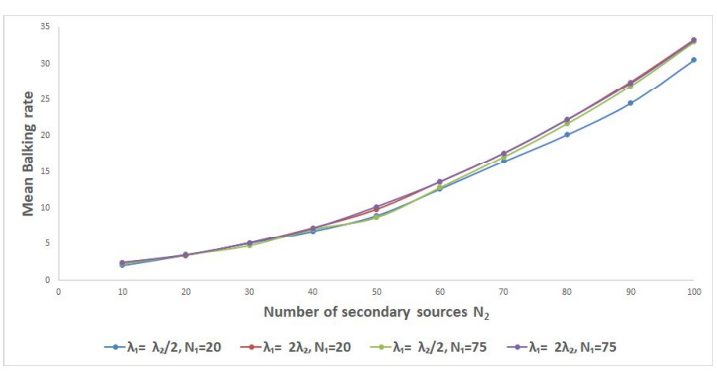

Figure 7. The effect of the primary network parameters on the mean balking rate of SUs vs $N_{2}$

Figure 8 depicts the effect of the secondary server's unreliability on the expected response time while the mean failure rate $\left(1 / \gamma_{1}\right)$ is increasing. Increasing the secondary mean failure rate, as expected, results in a longer response time for the affected customers. Figure 9 depicts the effect of the server 
unit's unreliability on the SUs' mean sojourn time. It shows the difference in the value of the mean response time as the mean repair rate increases $\left(1 / \gamma_{2}\right)$. As seen in this graph, having a reliable servers in CRN leads to a lower response time.

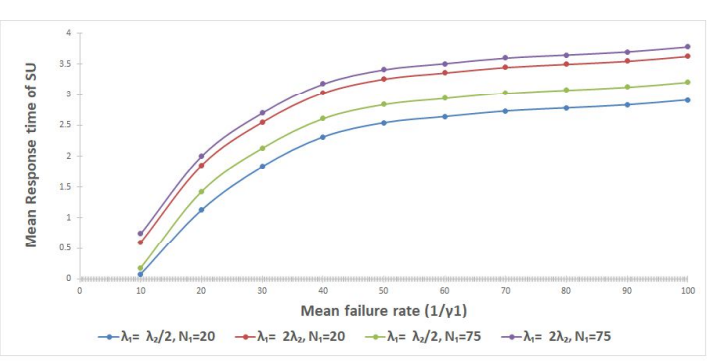

Figure 8. The influence of the non-reliability of the SCS on the secondary mean response time vs mean failure rate $1 / \gamma_{1}$

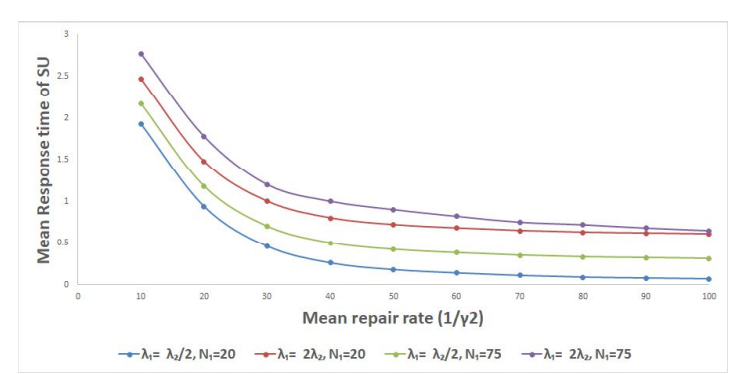

Figure 9. The impact of the non-reliability of the SCS on the mean sojourn time of the SUs vs mean repair rate $1 / \gamma_{2}$

\section{CONCLUSiON}

A finite-source retrial queuing system that contains two nonindependent parts was introduced in this paper. Our system was designed to simulate a cognitive radio network with primary and secondary service units with balking and reneging on the second part assuming that the later server is unreliable. A thorough review was carried out using simulation to investigate the effect of the service times distributions and the impact of the cognitive technology on the key performance measures of the system.

\section{ACKNOWLEDGMENT}

The research work of János Sztrik is supported by the EFOP-3.6.1-16-2016-00022 project. The project is co-financed by the European Union and the European Social Fund. The research of Mohamed Hedi Zaghouani is supported by the scholarship of Stipendium Hungaricum.

\section{REFERENCES}

[1] Wang L. C. Adachi F. Load-balancing spectrum decision for cognitive radio networks: IEEE Journal on Selected Areas in Communications. 2011. pp. 757-769.

[2] Devroye N., Vu M., Tarokh V. Cognitive radio networks: CIEEE Signal Processing Magazine 25.6. 2008. pp. 12-23.

[3] Gunawardena S., Zhuang W. Modeling and Analysis of Voice and Data in Cognitive Radio Networks: Springer. 2014.
[4] Nemouchi H., Sztrik J. Performance Simulation of Finite-Source Cognitive Radio Networks with Servers Subjects to Breakdowns and Repairs: Journal of Mathematical Sciences. 2019. pp. 702-711.

[5] Akyildiz I. F., Lee W. Y., Vuran M. C., Mohanty S. NeXt generation/dynamic spectrum access/cognitive radio wireless networks: A survey: Computer networks 50.13. 2006. pp. 2127-2159.

[6] Mitola J., Maguire G. Q. Cognitive radio: making software radios more personal: IEEE personal communications 6.4. 1999. pp. 13-18.

[7] Zaghouani M. H., Sztrik J. Performance simulation of finite-source Cognitive Radio Networks with impatient calls in the orbit: XXXVI International Seminar on Stability Problems for Stochastic Models. 2020

[8] Wuchner P., Sztrik J., De Meer H. Finite-source M/M/S retrial queue with search for balking and impatient customers from the orbit: Computer Networks. 2009. 53.8. 1264-1273.

[9] Kumar R., Som B. K. An M/M/1/N queuing system with reverse balking and reverse reneging: Advanced Modeling and Optimization. 2014. 339353.

[10] Fishwick, P. A. Simpack: getting started with simulation programming in $\mathrm{C}$ and $\mathrm{C}++$ : Proceedings of the 24th conference on Winter simulation. 1992. pp. 154-162.

[11] Zaghouani, M. H., Sztrik, J., and Uka A. Reliability Analysis of Cognitive Radio Networks: Annales Mathematicae et Informaticae. 2020. 52. pp. 255-265.

[12] Zaghouani, M. H., Sztrik, J., and Melikov A. Reliability Analysis of Cognitive Radio Networks: International Conference on Information and Digital Technologies (IDT). IEEE (2019). 557-562.

[13] Zaghouani, M. H., Sztrik, J., and Uka A. Simulation of the performance of Cognitive Radio Networks with unreliable servers. Annales Mathematicae et Informaticae. 2020. 52. pp. 255-265. 